\title{
Design of Information Management Platform for Laboratory Construction Based on Cloud Computing
}

\author{
Fang Liu ${ }^{a}$, Yingji Luo ${ }^{b}$, Ziyuan Gao ${ }^{c}$, and Jianhua Zhang ${ }^{d}$ \\ National Demonstration Center for Experimental Economics and Management Education(Guangxi Universit \\ y of Finance and Economics), Daxuexi Road NO.189,Nanning,Guangxi,China \\ aliuf319@gmail.com, b421895997@qq.com, ‘996123523@qq.com, d191792427@qq.com
}

Keywords: information management platform; laboratory construction; cloud computing technology

\begin{abstract}
The laboratory construction of the university provides support services on experimental and practical teaching for all majors through the integration and optimization of experimental resource allocation. By analyzing the current situation of laboratory information construction, this paper proposes to establish a laboratory construction information management platform by using cloud computing technology, and on this basis, design the functional structure and overall architecture of the platform.
\end{abstract}

\section{Introduction}

As an important place for colleges to carry out experimental teaching, scientific research and social services, the laboratory is an important place for cultivating students' practical ability, innovative ability and improving students' comprehensive quality. In recent years, with the continuous advancement of education and science and technology, it is necessary to strengthen the construction of laboratory information to improve the level of university laboratory construction. It has important significance in taking advantage of experimental resources, cultivating students' innovation and practical ability, and creating good social benefits.

National Demonstration Center for Experimental Economics and Management Education undertakes the task of optimizing and integrating the teaching software and hardware resources of the whole university. However, it has not made detailed plans for laboratory information construction, resulting in the annual use of more than half of the instruments and equipment. Although in recent years, unremitting efforts to how to strengthen the exploration and research of laboratory management, and how to improve the use efficiency. But this situation has not changed much. This paper builds a laboratory construction information management platform based on cloud computing technology, which can improve information management of university laboratories, work efficiency and management level.

\section{Current Situation of Laboratory Information Construction}

With the rapid development of technology, the information management method has penetrated into various industries. The construction of university laboratories can realize the informatization of open experimental teaching, which must meet the needs of information development of current and future development. Only in this way, students' Hands-on, practical ability and comprehensive quality are improved. At present, colleges and universities are increasingly investing in the management of laboratory equipment [1]. It has also played a positive role in promoting the construction of laboratory management information. However, there are inevitably many shortcomings in the process of use, mainly in:

Difficulties in collecting front-end information data. In the case of software system management-led, the laboratory's use process data, equipment usage value, equipment inventory and verification need to be manually entered. Equipment data procurement is very difficult, because no 
equipment is matched with the computer.

Not timely on Device data update. The usage data of the device is manually collected and entered. It takes a long time to collecting, entering and becoming effective of the data. This situation causes inability to grasp and query device information in a timely manner.

Increasing on the artificial consumption. The number of students in the school is constantly expanding, and the total number of laboratories is increasing, but the number of laboratory management personnel has not increased. This situation results in a heavier burden on laboratory managers.

Unreasonable on the management mode. The laboratory management mode follows the traditional management mode, which can not arrive effective supervision to the process of various experiments, and instant information management method. In the laboratory security management, it also lacks real-time informational early warning method.

Routine experimental management problems. It includes turning on the power freely, losing the borrowing easily, using collapsed equipment inconveniently, wasting resources on not closed equipment, and lacking measures on prevention of anti-fire and anti-theft.

\section{Design Goals of Laboratory Management Platform}

The laboratory management platform aims to solve the current situation of laboratory information construction, provide users with convenient and concise services and functions, improve resource utilization, computing speed and information security, and reduce equipment costs. It integrated most data and management transactions involved in laboratory management, the main goals are:

Supporting fixed laboratory information management. It supports laboratory-related management of course, personnel and equipment, meets the sharing of high-quality teaching resources, and builds a long-term laboratory management system in the future.

Supporting the needs of various users. It meets the practical needs of students, teachers and laboratory administrators for the laboratory, even the practical needs of many types of users such as laboratory asset managers and technicians.

Sharing experimental resource. It realizes the integration of different resource terminals, servers and various storage devices in the laboratory, provides massive data storage, management, sharing and access services, and supports virtual application access of different resource terminals.

Dispatching and distributing resources rapidly. It can manage the laboratory infrastructure, extract applications with high usage rate, realize the sharing of software and hardware resources at the application level, and quickly adjust and manage when the resource requirements of each laboratory change.

Simplifying operation: The platform provides a simple operation interface and convenient operation method. During the use process, the user pays more attention to the specific business functions and provides the application value of the platform.

\section{Demand Analysis}

Laboratory management platform networked laboratory management, experimental teaching management, instrument and equipment management, low-value products and consumables management, practice management, data and reporting, and experimental security management.

Including the whole process management of the use of laboratories, instruments and equipment, reducing the workload on laboratory management, improving work efficiency and external service level; strengthening the macro control of laboratory equipment information collection, material management, project construction, in order to save costs and improve utilization; strengthening open sharing of laboratories, breaking the boundaries between schools and schools, and fully sharing the resources of open laboratory laboratories; providing detailed data basis for equipment management departments to manage, supervise and control the entire laboratory, and effectively improving investment efficiency; monitoring and managing effectively personnel and equipment safety during 
the experimental process, increasing response and handling of emergencies, and retaining data basis; energy consumption in laboratory, and saving energy while ensuring safe use; monitoring timely of the experimental environment, timely response to the abrupt environment to ensure the safety of equipment and personnel; providing raw materials and basic data for laboratory teaching evaluation, laboratory construction and experimental teaching quality management.

\section{Design of Information Management Platform Based on Cloud Computing}

\subsection{Cloud computing technology}

There are three layers in the cloud computing architecture that are horizontal, namely the application layer, the platform layer, and the infrastructure layer [2, 3].

The infrastructure layer is mainly responsible for preparing the computing and storage resources required by the middleware layer or the user, such as resource virtualization and distributed storage.

The platform layer is mainly responsible for providing related software and hardware resources for system development. It can provide a variety of services based on the infrastructure layer, such as cache services and parallel processing, which can be used to support the display layer, or directly called by the user.

The application layer is mainly responsible for providing users with relevant. The application uses a variety of services provided by the middleware layer. The cloud computing is shown in Fig.1.

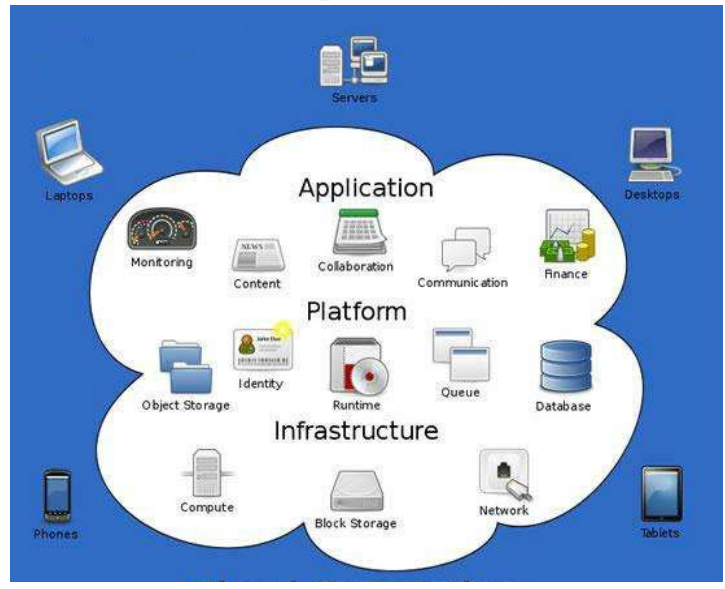

Fig.1. cloud computing
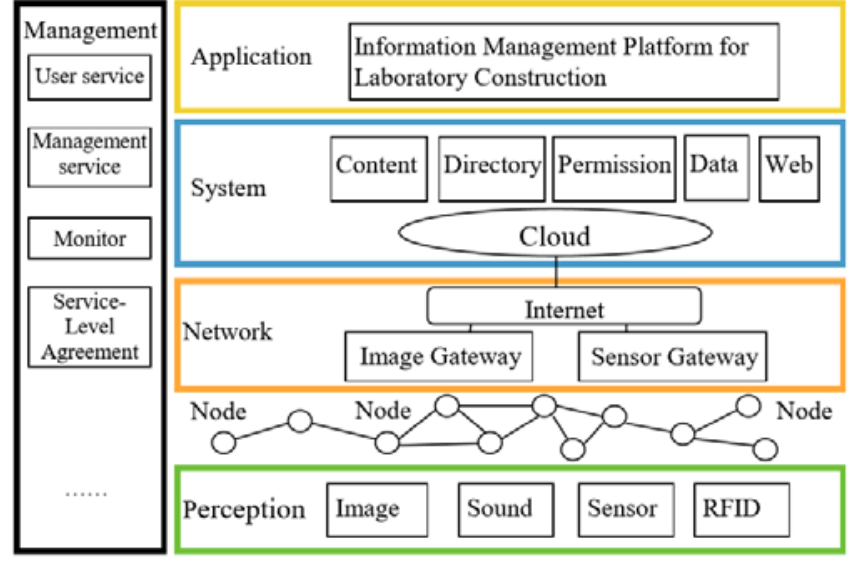

Fig.2. cloud architecture

\subsection{Design of cloud architecture}

The architecture is the basis for the operation of the laboratory information management platform. This platform integrates laboratory software and hardware resources and teaching resources with the concept of layered design to realize university management and open sharing, through combining cloud computing architecture system and laboratory management requirements, using the technology of distributed storage and server virtualization. The architecture of cloud platform is divided into four layers: the perception layer, the network layer, the system layer, and the application layer [4]. The architecture is shown in Fig. 2.

The perception layer's main equipment is the information collection and control node. The administrator sets the perception layer through software. The device can automatically collect laboratory information, such as lighting control, access control, power-on authorization control etc.

The network layer is the channel for transmitting control commands, which can be completed through the campus network transmission channel. It consists of a switch, a router, and a network cable.

The system layer is the data service center of the whole system. It completes the data analysis, processing, storage and remote service functions collected by the perception layer. The hardware devices include server, memory and system service software etc. 
The application layer is the interface of human-computer interaction, that is, the software system. The user sets the intelligent control of the system through software. The software system can realize multi-terminal information push display of mobile phones, tablets and computers.

\subsection{Platform architecture}

The cloud platform, for online office and business process related laboratory matters, can strengthen process supervision and scientific evaluation of business processes, and carry better management realization and management concepts. Also, it makes laboratory managers not worry data sharing, transactional verification and business processing between multiple systems. The platform can be a good assistant for laboratory management. The architecture is shown in Fig. 3.

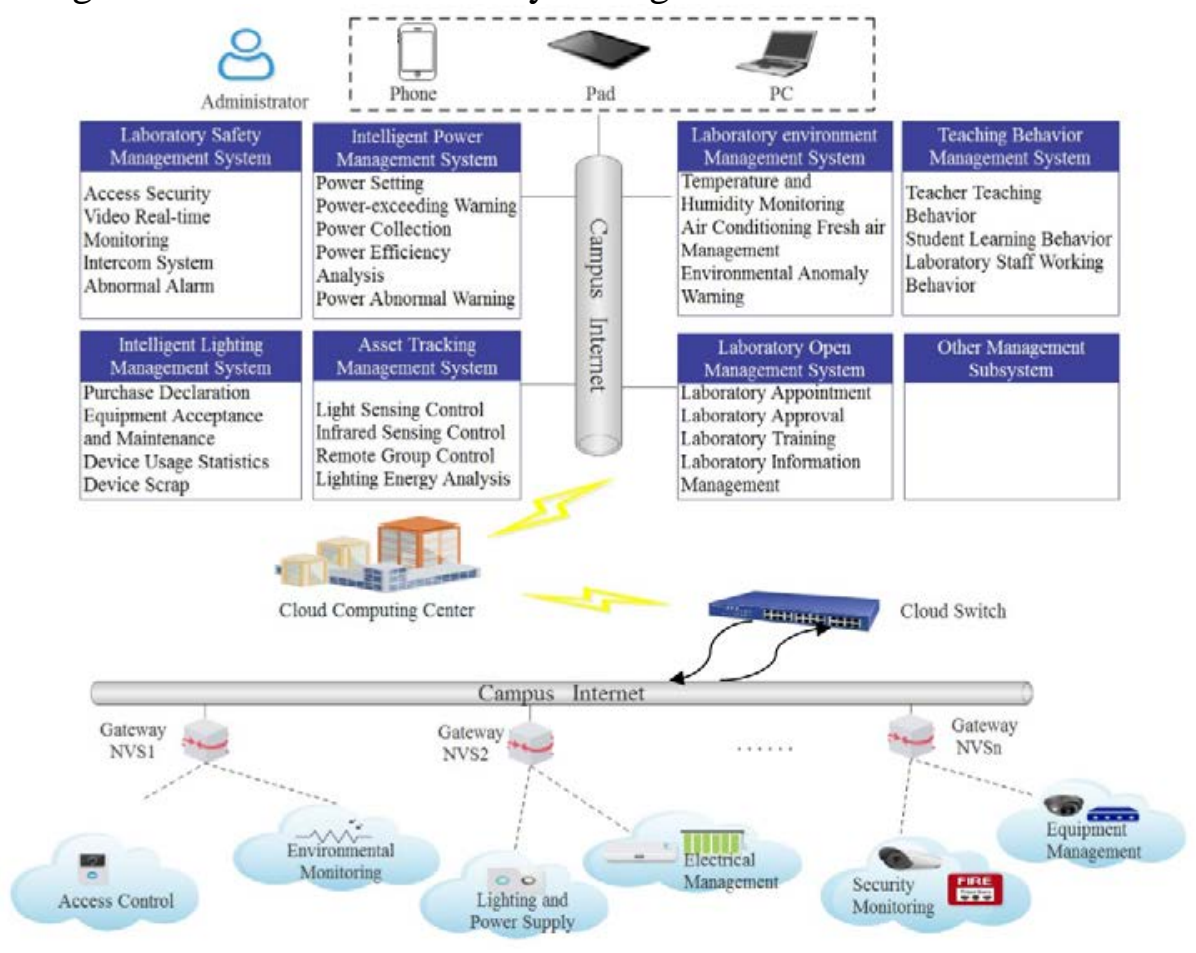

Fig.3. platform architecture

\subsection{Functional design}

The laboratory safety management system is mainly composed of access security control, video monitoring, infrared monitoring, intercom function and curtain control etc. It can control the safety of laboratories and equipment, and can make timely alarms for emergency events and save data.

The intelligent power management system is mainly composed of power control, data information management and alarm management, which enables effective control of the laboratory power supply and remote control. At the same time, it can count and analyze each laboratory electricity data, generate reports, remind the over-used laboratory, and alert the abnormal situation.

The intelligent lighting management system is mainly divided into two modes: automatic control and remote group control. The automatic control is divided into sound and light control and infrared control, and the lighting energy consumption is analyzed.

The asset tracking management system adopts a combination of software and hardware to automatically collect, process, and automatically collect statistics on the inventory, access, and usage rate of laboratory equipment. If the equipment is used illegally or brought out, the system will automatically alarm and remind you in real time to realize the real-time control.

The laboratory environmental management system mainly monitors the temperature and humidity, power distribution, air conditioning fresh, abnormal alarm, safety, energy measurement of the laboratory, which can alert the abnormal situation.

The teaching management system includes teaching behavior management and laboratory open management, which mainly includes participant behavior monitoring, laboratory appointment and 
use, experimental teaching management and security management etc. Through the combination of software and hardware, the system realizes sharing of data concentration management and collecting, pushing, displaying real-time information.

\subsection{Network structure mode}

The platform is made up of $\mathrm{B} / \mathrm{S}$ architecture [5,6]. For the daily management of the university, the system administrator needs to operate and manage multiple clients. The software of the B/S architecture only needs to manage the server. All the clients of the B/S architecture are browsers. The $\mathrm{B} / \mathrm{S}$ architecture has strong scalability, and it is more convenient to integrate between heterogeneous systems. All the software and the data required for the platform is installed on the server.

For applications with high concurrency (ten thousands of concurrent users), there may be more than one server hardware. To achieve clustering, we need a corresponding software supporting cluster, such as several server clusters as application servers, and several server clusters as database servers. Centralized storage management of data makes data more real-time, secure, unified and complete. The B/S structure is shown in Fig.4.

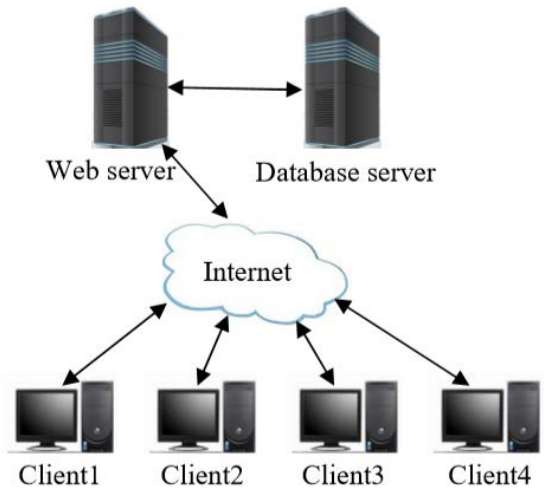

Fig.4. B/S structure

\section{Conclusion}

This paper innovatively uses cloud computing technology and combines the functional requirements to building the platform. The laboratory manager can only achieve unified management of the different laboratory through the cloud computing information platform. It plays an important role in the centralized management, software and hardware resources, experimental video library, case teaching network resource library and other experimental teaching and virtual simulation experimental teaching resources.

\section{Acknowledgements}

This work was financially supported by the Guangxi University of Finance and Economics Education and Teaching Reform Project (2018B22).

\section{References}

[1] Liu Fang, Luo Yingji, Zhang Jianhua. A Probe into the Construction and Standardized Management of Laboratory in University. Theory and Practice of Education. Forum Vol.36 (2016), P.15-16. In Chinese.

[2] YANG Yi. Research on Key Technologies of Intelligent Community Management Platform Based on Cloud Computing. Automation \& Instrumentation (2017), P.104-107. In Chinese.

[3] WU Xu, CHEN Ren-an, WEI Dezhi. Design and Application of Laboratory Integrated Management System under Cloud Computing. Research and Exploration in Laboratory. Forum Vol. 
34(2015), P.226-229. In Chinese.

[4] HENG Jun, WANG Xin-ning, HENG Hui. Design of the laboratory management information system based on cloud computing. Automation \& Instrumentation (2017), P.65-66, 69. In Chinese.

[5] Zhang Qian, Li Pan. Design of Laboratory Management Information System Based on Cloud Computing. China Computer \& Communication (2018), P.57-58. In Chinese.

[6] WU Yan-qing. Design of a Laboratory Management Information System Based on Cloud Computing. Research and Exploration in Laboratory. Forum Vol.32 (2013), P.291-296. In Chinese. 